\title{
Formation initiale et appropriation des compétences professionnelles par les enseignants stagiaires en éducation physique et sportive (EPS).
}

\author{
Maher Mrayeh ${ }^{1,}$ Ghislain Carlier ${ }^{2,}$ Youssef Feki ${ }^{3}$ \\ Institut supérieur de Sport et de l'Education physique de Ksar Said, Tunisie \\ Institut d'éducation physique et de réadaptation (IEPR)/ Université catholique de Louvain (UCL)/ Unité \\ éducation par le mouvement (EDPM) \\ Institut supérieur de Sport et de l'Education physique de Ksar Said, Tunisie Maher Mrayeh
}

\begin{abstract}
Summary: The purpose of this article is to assess the quality of teacher training in physical education and sport in Tunisians ISSEP. This assessment is based through the qualitative and quantitative analysis of the degree of mastery of professional skills. The observation focused on teaching 100 PES sessions. Five skills were set for this study : communication (1), the design of teaching situations (2), the management of situations of teaching (3), the assessment of learning (4) and the ethically and responsibly act (5). These skills were evaluated through criterion indicators. The results show that (skill.5) is closest to the total control. Mixed are the averages obtained in the first and the third skills. Finally, the second skill was the less well controlled.

Keywords: Initial training, appropriation, professional skills, teacher trainees, physical education and sports.
\end{abstract}

\section{Introduction}

Les instituts de sport et de l'éducation physique Tunisienne (ISSEP) poursuivent leur rénovation pédagogique dans le cadre des évolutions institutionnelles. C'est un facteur de changement des pratiques pédagogiques qui s'appuie néanmoins sur un modèle de formation bien ancré à l'école, celui qui privilégie la formation aux compétences et qui ne se limite pas à une transmission de savoirs académiques. L'analyse des objectifs professionnels ou d'apprentissage assignés à chaque module en témoigne. Les compétences peuvent être génériques: analyser et synthétiser, apprendre, résoudre des problèmes, utiliser ses connaissances dans la pratique, s'adapter à des situations, se soucier de la qualité, etc., ou elles peuvent être sectorielles et techniques. Un des enjeux de la formation à la compétence dans le contexte des formations statutaires ou des formations continues pour les cadres pédagogiques destinés pour l'enseignement porte prioritairement sur les compétences génériques. En outre, la compétence est toujours référée à l'activité et à un contexte (pas exclusivement professionnel), elle met en oeuvre tous types de savoirs et de modes de régulation de l'action : la compétence permet d'agir et de résoudre des problèmes professionnels de manière satisfaisante dans un contexte particulier, en mobilisant diverses capacités de manière intégrée (Bellier, 2000). C'est par l'analyse du travail et des interactions sociales que les compétences sont repérables. Finalisées, opérationnelles, apprises, tacites ou explicites, elles expliquent la performance perçue (Samurcay et Pastre, 1995). Le défi à relever pour l'école consiste aujourd'hui à proposer d'acquérir des compétences professionnelles de qualités. Il s'agit de faire converger les exigences réglementaires de validation des acquis de l'expérience, les enjeux sur les formations professionnelles diplômantes dans le domaine de l'enseignement de l'éducation physique et sportive (EPS) et la modernisation de la fonction publique enseignante tunisienne.

La politique de recherche et de développement pédagogique engagée par les ISSEP vise à accompagner ces changements organisationnels et pédagogiques. Dans ce cadre, nous observons que le recours à une ingénierie de la compétence et du renforcement professionnel (Gomez, 2004) butte sur un obstacle, celui d'un inachèvement de la construction des référentiels de compétences et de positionnement à l'entrée en formation. L'objectif est néanmoins clairement énoncé, l'utilisation de ces référentiels de compétences déclinés ensuite en référentiels de formation puis référentiels d'évaluation doit engendrer des pratiques d'individualisation de la formation et de développement de l'autoformation (Carre et Caspar, 2002), y compris et notamment, en contexte institutionnel (Albero, 2000).

L'approche pédagogique se trouve ainsi renouvelée : tout l'effort d'apprentissage ne saurait résulter simplement de l'action des enseignants (Vygotski, 1997), l'acquisition de compétences professionnelles résulte aussi de l'autoformation du sujet apprenant, c'est-à-dire de l'autodirection de ses apprentissages et de sa double dimension motivationnelle et autorégulatrice. La première, proactive, consiste à s'orienter vers des buts de formation et à sélectionner des situations d'apprentissage possibles. La deuxième, métacognitive, s'intéresse au contrôle du sujet sur ses modes d'apprentissages (Carre et Moisan, 2002). Les transformations rapides des sociétés nécessitent l'acquisition de nouvelles compétences chez les enseignants et, dans le même temps, de 
nouvelles fonctions leurs sont dévolues. Une nouvelle professionnalité enseignante est en voie d'émergence. Le nouveau rôle des enseignants d'EPS nécessite alors une formation pédagogique approfondie, mais aussi des formations à la gestion de l'hétérogénéité des classes, tant du point de vue des milieux sociaux d'origine des élèves ainsi que leurs aptitudes cognitives et motrices. Les enseignants doivent nécessairement maîtriser les nouvelles technologies, individualiser leur intervention, développer l'autonomie chez les élèves et le travail en partenariat. Comment sont formés les futurs enseignants à leurs nouveaux rôles, aux évolutions des savoirs scientifique et méthodologique aux besoins des élèves en perpétuelle évolution ? Les modèles du praticien réflexif ou de l'organisation apprenante sont-ils pertinents pour former les enseignants d'EPS ? Quels sont les critères qui définissent l'enseignant d'EPS efficace ? Quelle place est donnée aux différents types de savoirs et à l'acquisition de compétences sociales et instrumentales dans la formation des enseignants ? Quelles sont les perspectives souhaitables pour l'avenir ? Il n'en reste alors l'idée de vouloir identifier les apports de la formation initiale à travers la qualité d'appropriation des compétences professionnelles en matière d'intervention pédagogique en sciences et techniques des activités physiques et sportives.

\subsection{Evaluation de la formation initiale des enseignants}

\section{Contexte théorique}

La question de l'évaluation des formations professionnelles universitaires (Bourdoncle et Lessard, 2003) semble peu explorée que ce soit dans le domaine de l'évaluation des acquis des étudiants (Romainville, 2002) ou dans celui de l'évaluation de l'influence des dispositifs de formation et des cultures disciplinaires sur la construction des compétences professionnelles dans le cadre de la formation initiale des enseignants du primaire et du secondaire (Brau-Antonny, 2008).

En effet, les recherches centrées sur les compétences et savoirs professionnels construits en formation initiale par les enseignants sont donc peu développées. On sait finalement peu de choses sur le processus par lequel passent les enseignants stagiaires pour apprendre un métier dont la complexité n'a fait que croître depuis une vingtaine d'années. En ce qui concerne l'évaluation des acquis de la formation initiale dispensée aux différentes institutions universitaires de formation des cadres pédagogiques, les études sont également peu nombreuses. Nous laisserons volontairement de côté les écrits qui condamnent de façon caricaturale les orientations de la formation professionnelle des enseignants dans ces institutions, au profit de recherches plus rigoureuses (Rayou et Van Zanten, 2004 ; Rayou, Gélin et Ria, 2007) permettant d'avoir une vision plus éclairée du jugement que portent les jeunes enseignants sur leur formation. Ces travaux montrent que le principal grief exprimé par les enseignants stagiaires à l'encontre de la formation initiale concerne l'aspect trop théorique d'une formation perçue comme étant souvent éloignée des conditions d'exercice du métier.

Toutefois, il est à signaler que dans la plupart du temps, les évaluations des actions de formation fournissent une production foisonnante d'informations sur le déroulement de ces actions sans qu'il y ait intervention explicite des objectifs. Selon Guskey (2000), les pratiques d'évaluation les plus courantes se contentent d'utiliser des questionnaires de satisfaction des participants sans que soit véritablement mis en évidence ce qu'ont appris les enseignants. Alors que l'évaluation devrait rendre compte des changements à venir dans les pratiques des enseignants et se rapporter à leur développement professionnel, elle n'accorde finalement que peu d'importance à des indicateurs de transformation de leur activité et de leur profil compétentiel. Ainsi l'utilisation par les participants de nouvelles connaissances et compétences acquises au cours de leur formation devrait constituer l'enjeu principal d'une évaluation de la formation professionnelle, cette évaluation pouvant même déboucher sur les effets produits sur les résultats des élèves (Day, 2004). Évaluer des compétences développées en formation soulève de nombreuses difficultés méthodologiques car il convient d'identifier des indicateurs rendant compte d'une modification des pratiques. Huberman (1992) signale que c'est une opération délicate qui suppose une observation prolongée de l'activité enseignante et qui oblige l'observateur à construire toute une batterie d'indicateurs notamment des indicateurs conceptuels qui renvoient aux idées et aux représentations et des indicateurs instrumentaux qui sont relatifs à l'intervention de l'enseignant quand il est face à ses élèves. Par ailleurs, quand on tente d'évaluer les effets de formation chez les enseignants on remarque qu'ils sont très fluctuants (Cauterman, Demailly, Suf-fys et Bliez-Sullerot, 1999). Ces changements peuvent être très éphémères voire inexistants, on note également des écarts importants entre les intentions affichées par les formateurs et ce que retiennent réellement les enseignants (aspect déductif).

Enfin une action de formation ne constitue pas toujours la variable déterminante pouvant expliquer une transformation des pratiques professionnelles. L'autoformation, l'expérience personnelle mais aussi le partage de l'expérience avec les pairs peuvent aussi jouer un rôle moteur dans la construction des compétences professionnelles. Malgré ces nombreux obstacles faut-il pour autant renoncer à évaluer la capacité qu'a l'enseignant à mobiliser, en situation de travail, les connaissances et compétences acquises en formation ?

Donner la possibilité aux bénéficiaires d'une formation professionnelle d'évaluer la qualité de leur formation, c'est d'abord être capable de clarifier le référent de l'évaluation (Barbier, 1985 ; Hadji, 1997) c'est-à-dire ce par rapport à quoi il est possible d'émettre un jugement de valeur. La définition des critères d'évaluation constitue 
donc l'axe central autour duquel doit se structurer toute évaluation de la formation, ce qui nécessite de déterminer la cible à atteindre (à quel métier forme-t-on ?). Évaluer la formation suppose donc de préciser le plus finement possible le métier auquel les instituts supérieurs de formation des cadres pédagogiques est censé former, d'en cerner les contours en dégageant ce que l'on est en droit d'attendre d'un enseignant quand il exerce son métier. Cette réflexion préalable impose donc aux concepteurs de ce type d'évaluation de bien cerner les compétences professionnelles visées. Nous partons du postulat que le métier d'enseignant possède une identité structurée autour d'un noyau stable de compétences que doit mobiliser chaque enseignant quel que soit le contexte d'exercice. Ces compétences s'appuient sur des valeurs et des représentations du métier partagées et participent à l'insertion des acteurs dans un groupe professionnel bien identifié, ce qui ne veut pas dire que l'on ignore l'existence de cultures professionnelles spécifiques. Nous avons ainsi dégagé quatre domaines de compétences professionnelles : la conception et planification de l'enseignement, la gestion de classe et régulation des apprentissages, l'analyse réflexive et autoévaluation et la responsabilité et l'éthique professionnelles.

Penser la professionnalité enseignante en termes de compétences nous permet dès lors de considérer les différents dispositifs de formation comme un ensemble de moyens destinés à enclencher chez les enseignants stagiaires un processus de construction de compétences.

\subsection{La didactique professionnelle pour analyser les pratiques d'enseignement}

D'après les écrits de Maubant (2007), il est d'usage aujourd'hui de considérer que différents dispositifs ou démarches peuvent soutenir, promouvoir et faciliter la professionnalisation des enseignants. Qu'il s'agisse des dispositifs de formation par alternance, des dispositifs d'analyse des pratiques ou encore des démarches par problèmes, les organismes de formation professionnelle s'intéressent de plus en plus aux processus facilitateurs de la professionnalisation. Or, il semble que les recherches portant sur la professionnalisation ont peu appréhendé jusqu'à présent la dimension didactique de l'apprentissage professionnel. Il est donc nécessaire de montrer en quoi le cadre théorique proposé par la didactique professionnelle constitue une perspective pertinente pour accompagner l'apprentissage professionnel, en particulier parce qu'il propose de lire et de comprendre la pratique d'enseignement, point de départ de l'esquisse d'une théorie de la pratique enseignante.

En effet, la finalité intrinsèque de la professionnalisation est bien la recherche d'une rationalité instrumentale conduisant à la professionnalité (Lenoir et Vanhulle, 2006). Celle-ci peut être définie comme la capacité du professionnel à mettre en oeuvre de manière rationnelle les savoirs nécessaires à l'exercice de la profession. Dans cette perspective, l'institution de formation cherchera à identifier les processus facilitateurs de l'apprentissage professionnel. Dès lors, la lecture compréhensive de l'activité d'enseignement, qui peut prendre plusieurs figures, en particulier celle constitutive de démarches d'analyse des pratiques, devrait contribuer à faciliter l'apprentissage professionnel (Brown, JS.S et Duguid, P. 1989). Si l'on peut considérer que la condition pour réussir le processus de professionnalisation est bien la centration de la formation sur l'apprentissage professionnel, le cadre théorique offert par la didactique professionnelle (Pastré, 2002 ; Habboub et Lenoir, 2005) peut constituer un cadre pertinent d'analyse et de compréhension de l'acte professionnel.

\subsection{La didactique professionnelle pour comprendre l'acte d'enseignement}

L'approche par compétences cherche à favoriser une meilleure compréhension des réalités du monde, au service d'un agir social pour l'élève et d'un agir socio-professionnel pour l'enseignant. Pour ce dernier, l'apprentissage et la maîtrise des douze compétences au service d'un savoir-agir professionnel constituent le cadre de la démarche de professionnalisation (MEQ, 2001).

Dans l'esprit de la réforme de l'école, l'enseignant est donc bien un apprenant, au sens où l'apprentissage proposé vise la construction et la maîtrise d'un savoir-agir professionnel incarné par les douze compétences. Dès lors, il est rappelé aux institutions en charge de la formation des enseignants de tout faire pour créer les conditions les plus favorables à cet apprentissage devant faire sens pour l'enseignant en formation, car étant pensé et mis en oeuvre au service d'un agir professionnel en construction. Cette finalité d'un " agir professionnel » à construire définirait et justifierait en quelque sorte l'approche par Certes, le concept de compétence peut constituer l'apanage d'un discours officiel incantatoire et prescriptif visant l'adaptabilité socioprofessionnelle. Mais il n'est en aucun cas opératoire en termes de compréhension de l'activité d'enseignement. Il semble donc essentiel de revenir à la problématique des savoirs, et donc de recourir aux modèles proposés par la didactique professionnelle.

Il convient, à l'instar de Clancey, W. J. (1991) de considérer qu'il n'y a pas de savoir sans compétence ni de compétence sans savoir. Astolfi (2003) poursuit cette idée: «Posséder un savoir, ce n'est pas seulement le mémoriser, c'est s'en servir d'outil avec compétence. Quant à la compétence qui ne disposerait pas d'un savoir pour l'éclairer, elle ne serait qu'une recette grâce à laquelle on réussit sans comprendre». Cette réserve majeure faite au concept de compétence explique sans doute pourquoi les recherches actuelles en didactique professionnelle semblent se recentrer sur la problématique des savoirs professionnels en jeu dans l'exercice de 
l'activité professionnelle (Pastré, 2002). Ce retour du savoir dans les débats scientifiques sur la formation professionnelle des enseignants peut expliquer aussi le glissement qui semble s'opérer aujourd'hui entre une didactique professionnelle et une didactique des savoirs professionnels (Habboub, Lenoir et Tardif, 2005). Outre la nécessité de se centrer sur l'identification des savoirs constitutifs de l'agir professionnel, Maubant, Dhahbi, J et Chouinard, I (2007) avaient pu montrer dans des travaux antérieurs que les recherches en formation professionnelle des adultes peinent souvent à identifier le système d'ensemble qui permet d'analyser et de comprendre l'articulation entre les composantes de l'apprentissage (les différentes formes des savoirs) et l'action même d'apprendre (Maubant, 2004). Les recherches sur la formation des enseignants se heurtent à la même impasse. S'il semble possible de repérer les différents savoirs en jeu dans l'activité professionnelle, il semble plus délicat d'identifier le système qui régit la pratique, ce que Bru (2004) qualifie de principes organisateurs de la pratique.

Comprendre l'agir professionnel requiert d'identifier ses éléments constitutifs mais aussi ce qui participe de leur opérationnalisation. Dès lors, nous pouvons admettre l'importance accordée aux recherches qui vont tenter de privilégier un triple regard : l'identification des savoirs professionnels, la compréhension des processus de construction et de mise en œuvre des savoirs professionnels dans l'exercice de la profession et l'identification des éléments constitutifs, contributifs et facilitateurs de l'apprentissage professionnel. Il semble donc important ici de proposer une rupture épistémologique dans la conception des dispositifs de formation des enseignants en privilégiant l'entrée par l'analyse de la pratique d'enseignement, celle-ci se fondant sur ce triple regard. Dès lors, la didactique professionnelle peut constituer un cadre référence pertinent pour analyser l'acte d'enseigner.

\subsection{Population}

\section{Méthodologie}

Cette étude porte sur un échantillon de 100 étudiants ( 65 hommes et 35 jeunes filles) âgés entre 24 et 25 ans, soit $37 \%$ de l'effectif total des étudiants inscrits dans les classes terminales de la filière Maîtrise en EPS dispensée dans les ISSEP de Tunis, de Sfax et du Kef. Aucun de ces étudiants n'a eu une expérience professionnelle dans un club civil ou scolaire en matière de pratique sportive, en tant que joueur ou en tant qu'entraîneur, durant son cursus de formation universitaire. A cet égard, 100 séances d'éducation physique destinées à des classes mixtes de $7^{\text {ème }}$ et $8^{\text {ème }}$ année de base ayant un effectif de 25 à 30 élèves par classe ont fait l'objet d'une observation didactique. Ces séances ont été consacrées à l'enseignement des activités physiques et sportives (APS) suivantes :

\section{Insérer le tableau no 1 ici . 3.2 Instrumentation}

Les données recueillies portent sur l'enregistrement vidéoscopique de toutes les interventions des étudiants stagiaires durant les cours dispensés. A cet occasion, deux caméscopes numériques (25 images en PAL) ont été utilisée, l'une placée sur le bord du terrain et qui recouvre la totalité du plateau de travail et l'autre utilisée par l'expérimentateur lui même .Le dispositif utilise le couplage son/image afin de pouvoir mettre en relation des comportements des différents acteurs et les dires de chacun (consignes, feedbacks individuels et collectifs). Pour se faire chaque enseignant observé a été bien équipé par un micro-baladeur branché à une table de mixage. L'évaluation a été effectuée par l'expérimentateur.

\subsection{Déroulement}

L'observation didactique des séances d'éducation physique et sportive menée a eu lieu juste avant l'évaluation finale des prestations des étudiants lors du stage de préparation à la vie professionnelle. Une période d'environ 55 minutes fut nécessaire pour que chacun des enseignants évalués puisse mener et assurer la conduite de sa séance à terme. Tous les enseignants stagiaires ont été filmés au moins pendant une séance avant l'enregistrement des données, dans le but d'habituer les protagonistes de l'étude au matériel utilisé. Afin de réduire les biais liés à l'effet Hawthorne (e.g., Adair et al., 1989) chez les enseignants (i.e., modification des comportements due à la présence d'un observateur), l'expérimentateur s'est présenté à l'enseignant comme étant étudiant menant une enquête sur la motivation des élèves en EPS, sans faire aucune allusion à l'effet Pygmalion.

\subsection{Considération éthique}

Les classes concernées et les enseignants stagiaires ont été filmés au moins une séance avant l'enregistrement des données. Cette opération a pour but d'habituer les protagonistes de l'étude au matériel utilisé et réduire ainsi l'effet Hawthorne (Adair, 1989) chez les enseignants. Pour le choix des compétences à observer, nous sommes partis du postulat que le métier d'enseignant possède une identité structurée autour d'un noyau stable de compétences que doit mobiliser chaque enseignant, quel que soit le contexte d'exercice (Marroy, 2005). Notre observation a porté sur cinq compétences de l'ensemble des 12 compétences constituants 
le référentiel de compétences largement étayées par les textes régissant la formation dans les ISSEP tunisiens et qui sont confondément précisées par l'ensemble des écrits scientifiques (Roux-Pérez, 2004). En effet, nous distinguons la compétence relative à la qualité de la communication dans les divers contextes liés à la profession enseignante (compétence 1), la conception des situations d'enseignement et d'apprentissage (compétence 2), le pilotage des situations d'enseignement et d'apprentissage (compétence 3), l'évaluation de la progression des apprentissages et le degré d'acquisition des compétences pour les contenus à faire apprendre (compétence 4) et la capacité des étudiants à agir de façon éthique et responsable dans l'exercice de leur fonction (compétence 5).

Chacune de ces compétences a été déterminée par cinq indicateurs qui lui sont spécifiques. Ces indicateurs ont été évalués par une échelle d'appréciation de Likert allant de 1 à 5 . En effet, avoir une moyenne de 5 points signifie que la compétence a été largement maîtrisé (TM), comptabiliser 4 points signifie que la compétence de l'étudiant stagiaire est clairement assuré (AS), avoir 3 points c'est la valeur moyenne et cela signifie que la compétence de l'étudiant stagiaire est acceptable (AC), avoir 2 points c'est dire que la compétence de l'étudiant stagiaire est peu développé (PD) et enfin avoir une moyenne égale à 1 signifie que la qualité de compétence est très peu développé (TPD)

\subsection{Fiabilité des codages et traitement statistique des données}

Les indicateurs relatifs à chaque compétence ont été codés par cinq observateurs spécialistes de l'intervention en EPS. Trois leçons aléatoirement sélectionnées ont été codées séparément par les cinq observateurs, sans qu'ils ne puissent se consulter. L'analyse des cœfficients kappa de Cohen (1960) et Bakerman et Gottman, (1997) a révélé une homogénéité des codages inter-observateurs comprise entre 0,60 et 0,80 pour les différents indicateurs spécifiques aux compétences choisies. L'analyse statistique des résultats a porté essentiellement sur le calcul de la moyenne, l'écart type, la variance ainsi que le calcul du pourcentage de réussite relatif au degré de satisfaction de chaque indicateur choisi.

\section{Résultats}

\subsection{Analyse de la qualité d'appropriation des compétences professionnelles Insérer le tableau no 2 ici}

Les données du tableau $\mathrm{n}^{\circ} 2$ indiquent que la capacité des étudiants stagiaires à agir de façon éthique et responsable (comp5) est très développés $(\bar{x}=15,63 \pm 2,28)$. Leurs performances obtenus au niveau de la compétence $1(\bar{x}=13,34 \pm 1,95)$, portant sur la clarté de la communication et celle de la compétence $3(\bar{x}$ $=13,11 \pm 2,36$ ) relative à la manière de piloter les situations d'enseignement-apprentissage abordées au moment de l'observation sont relativement développés. En revanche, la qualité de la compétence portant sur la conception des situations d'enseignement et d'apprentissage, est la moins développée par les stagiaires $(\bar{x}$ $=12,05 \pm 1,98)$.

\subsection{Analyse statistique des compétences évaluées}

4.2.1 Compétence 1 : communiquer clairement et correctement dans la langue d'enseignement, à l'oral et dans les divers contextes liés à la profession enseignante.

\section{Insérer la figure no 1 ici}

Les résultats affichés par la figure $n^{\circ} 1$, montrent qu'un bon nombre de stagiaires ont développé un niveau de communication qui satisfait minimalement aux exigences de la profession enseignante (26,6\% (acceptable) et $19,2 \%$ (assuré). Néanmoins, il est à signaler que 48,8\% des stagiaires ont développés des compétences communicationnelles en dessous du niveau optimum souhaité (peu développé). Enfin, 3,6\% des étudiants sont évalué en dessous ces normes (très peu développé).

\section{Insérer le tableau no 3 ici}

L'analyse tableau $\mathrm{n}^{\circ} 3$ permet de déduire que les prestations des stagiaires sont relativement satisfaisantes en ce qui concerne l'utilisation d'un langage varié dans différents contextes $(\bar{x}=2,78 \pm 0,86)$, la correction des erreurs commises par les élèves $(\bar{x}=2,81 \pm 0,85)$ et au niveau des échanges relatifs aux divers contextes liés à la profession enseignantes $(\bar{x}=2,54 \pm 0,80$ ). Néanmoins, certains de ces stagiaires éprouvent des difficultés relatives aux interventions pédagogiques auprès de leurs élèves $(\bar{x}=2,51 \pm 0,72)$.

4.2.2 Compétence 2 : concevoir des situations d'enseignement et d'apprentissage pour les contenus à faire apprendre.

\section{Insérer la figure no 2 ici}

L'analyse de la figure $\mathrm{n}^{\circ} 2$ révèle qu'un bon nombre de la population évaluée a pu assurer le minimum exigé pour l'appropriation de cette compétence $(29,80 \%$ (acceptable) et 14,20\% (assuré). En outre, des difficultés ont été enregistrées au niveau de la conception des situations d'enseignement et d'apprentissage pour les contenus à faire apprendre par un pourcentage assez élevé des stagiaires évalués (38,8 \% (peu dévelopé) et $17,20 \%$ (très peu développé)). 


\section{Insérer le tableau no 4 ici}

Le données illustrées dans le tableau ${ }^{\circ} 4$, indiquent que la majorité des étudiants observés ne prévoient pas dans leurs cours d'EPS des situations motrices de consolidation parallèlement à ce qu'ils ont prévu $(\bar{x}=1,92 \pm$ $0,97)$. En outre, il est à constater aussi, que ces futurs enseignants pratiquent à peine une pédagogie adaptée aux besoins particuliers de leurs élèves $(\bar{x}=2,47 \pm 0,83)$. Toutefois, nous avons constatés que la participation active des élèves dans les situations d'apprentissage ainsi que le respect de leurs champs d'intérêt sont moyennement pris en considération par l'ensemble des stagiaires observés $(\bar{x}=2,57 \pm 0,92)$. De même, ces résultats affichent que ces stagiaires éprouvent des difficultés à préciser les critères d'évaluation relatifs aux des objectifs pédagogiques abords $(\bar{x}=2,53 \pm 0,83)$.

4.2.3 Compétence 3: Piloter des situations d'enseignement et d'apprentissage pour les contenus à faire apprendre, et ce, en fonction du développement des compétences visées.

Insérer la figure no 3 ici

L'analyse des résultats obtenus (figure $n^{\circ} 3$ ) indique qu'un bon nombre parmi les étudiants observés a pu, à la fin du stage de la pratique pédagogique approprier l'habilité pédagogique relative au pilotage des situations d'enseignement-apprentissage pour les contenus à faire apprendre (31\% (acceptable) et 20,80\% (assuré) et 1,2 $\%$ (très maîtrisé)). Toutefois, il est à signaler que $47 \%$ des stagiaires ont éprouvé des difficultés à s'approprier cette compétence.

\section{Insérer le tableau no 5 ici}

L'observation didactique (tableau $\mathrm{n}^{\circ}$ 5) a révélé que les étudiants stagiaires s'assurent convenablement de l'organisation des élèves ( $\bar{x}=2,85 \pm 0,89)$, ils encouragent leurs élèves par des gestes discrets ou par des paroles lors de la réalisation des différentes tâches motrices $(\bar{x}=2,86 \pm 0,95)$ et enfin un bon nombre de stagiaire utilise la démonstration en tant que moyen didactique pour expliquer la tâche motrice à exécuter $(\bar{x}$ $=2,73 \pm 0,89$ ). Néanmoins, il à souligner que les interactions didactiques entre les stagiaires et leurs élèves nécessitent d'être mieux entretenues $(\bar{x}=2,24 \pm 0,99)$. Enfin, nous avons pu constaté que les stagiaires ont des difficultés à respecter les situations d'apprentissages préalablement prévues $(\bar{x}=2,43 \pm 1,12)$.

4.2.4 Analyse de la compétence $4:$ Evaluer la progression des apprentissages et le degré d'acquisition des compétences pour les contenus à faire apprendre.

\section{Insérer la figure no 4 ici}

Les valeurs statistiques illustrées ci-dessus (figure $\mathrm{n}^{\circ} 4$ ), montrent qu'un pourcentage assez important de stagiaires n'ont pas développé cette compétence (43,20\% (peu développé) et $11,20 \%$ (très peu développé)). Tout de même, 29\% des stagiaires ont relativement développés l'aptitude à évaluer l'état de progression des apprentissages et le degré d'acquisition des compétences pour le contenu à faire apprendre. Toutefois, nous notons que $14,20 \%$ de ces stagiaires ont pu clairement acquis cette compétence.

\section{Insérer le tableau no 6 ici}

Les données du tableau $n^{\circ} 6$, montrent les stagiaires n'arrivent pas à amener leurs élèves à expliquer respectivement leurs réussites ou leurs difficultés $(\bar{x}=2,34 \pm 0,81)$ et ne soutiennent pas suffisamment les initiatives personnelles de leurs élèves sur les démarches pédagogiques qu'ils s'approprient $(\bar{x}=2,36 \pm 0,97)$. De même, l'utilisation des moyens didactiques (grille d'observation, grille d'évaluation descriptive) destinés à l'évaluation de la progression des apprentissages proposés semble être à peine maîtrisé par ces stagiaires $(\bar{x}$ $=2,46 \pm 1,13)$. Toutefois, nous constatons que ces étudiants effectuent des rétroactions avec leur élèves en vue de leur permettant de prendre conscience de leurs forces et leurs attitudes $(\bar{x}=2,68 \pm 0,86)$. Ces feed-back sont également suivis par des bilans relatifs aux apprentissages effectués. Ces bilans conduis, par un nombre assez important des stagiaires $(\bar{x}=2,71 \pm 0,80$ ), portent des jugements sur les prestations quantitatives et qualitatives des élèves.

\subsubsection{Compétence 5: Agir de façon éthique et responsable dans l'exercice de ses fonctions.}

\section{Insérer la figure no 5 ici}

Les résultats illustrés par la figure $\mathrm{n}^{\circ} 5$ indiquent la compétence $\mathrm{n}^{\circ} 5$ est une habileté professionnelle appropriée par $71,40 \%$ des stagiaires, dont $10 \%$ ont excellé au niveau de l'exercice de leur fonction et se sont comportés de façon hautement responsable. Néanmoins, 28,60 \% de ces stagiaires n'ont pas acquis cette compétence, parmi eux $3,80 \%$ qui l'ont totalement raté.

\section{Insérer le tableau no 7 ici}

Le recours aux données du tableau $\mathrm{n}^{\circ} 7$ permet de constater que la majorité des indicateurs ont été largement assurés par l'ensemble des candidats observés, ainsi l'intégration sociale des enseignants stagiaires dans la vie institutionnelle semble être réussie. En effet, tous les étudiants observés maintiennent une attitude positive envers leurs collègues ( $\bar{x}=3,3 \pm 0,96$ ), ils agissent avec prudence contre toute forme de discrimination envers 
les élèves et d'une manière démocratique dans leur classe ou dans les ateliers de travail $(\bar{x}=3,16 \pm 0,95)$ et ils maintiennent une attitude positive envers tous leurs élèves $(\bar{x}=3,83 \pm 0,73)$.

\section{Discussion}

Les résultats obtenus dans cette étude ont montré que les étudiants des ISSEP Tunisiens achèvent leur cursus de formation universitaire avec un référentiel de compétence professionnel qui ne leur permet pas d'affronter le marché de l'emploi en qualité d'enseignant d'EPS (des difficultés au niveau de la capacité relative à la communication dans la langue d'enseignement, l'aptitude à concevoir et à piloter les situations d'enseignement et d'apprentissage, l'aptitude à évaluer la progression des apprentissages et le degré d'acquisition des compétences des élèves.) En effet, les difficultés liées à l'habileté communicationnelle se traduisent par une mauvaise portée de la voix, le vocabulaire utilisé n'est pas toujours en adéquation avec ce que peuvent comprendre les élèves et les métaphores ne sont pas toujours adaptées. Or nous savons bien que les activités physiques et sportives sont multiples et variées et la principale fonction didactique des instructions verbales réside dans la clarification du but et des sous-buts de la tâche (Gentile, 1972; Famose, 1983; Shiffrin et Schneider, 1977). Il s'agit sans doute là du principal apport de l'analyse de la tâche à la didactique des APS, audelà même de la problématique de l'aménagement du milieu. L'élève doit connaître avec la plus grande clarté possible l'objectif à atteindre. Une première distinction peut permettre de classer les habiletés en fonction du degré d'incertitude des sous-buts de la tâche: on parlera d'habiletés techniques lorsque buts et sous-buts seront certains, car uniquement déterminés par les contraintes réglementaires, environnementales et biomécaniques, et d'habiletés stratégiques lorsque la pertinence des sous-buts dépendra à tout instant de l'évolution de la situation, sous l'influence du sujet lui-même, de l'adversaire ou du milieu. Cette distinction peut expliquer certains résultats expérimentaux, qui peuvent, à première vue, sembler divergents. Ainsi Famose, Hébrard, Simonet et Vivès (1979) comparant dans le cadre de l'apprentissage d'habiletés techniques (ski, athlétisme) l'efficacité de méthodes basées sur la démonstration et l'explication d'une part, et sur l'aménagement du milieu d'autre part, montrent clairement l'avantage des secondes. La démonstration ou l'explicitation des moyens à mettre en oeuvre pour atteindre le but de la tâche, non seulement se révèlent peu pertinentes quand il s'agit de routines automatisées, mais encore risquent d'entraîner une confusion des buts: l'enseignant, en fournissant des informations et des consignes sur ce que doit être la forme du geste, rend par la même occasion le but de l'activité obscur au pratiquant (Gentile, 1972). Par contre, la clarification des buts et sous-buts de la tâche, verbalement et/ou par aménagement du milieu, permettraient aux élèves d'entrer dans un processus autoadaptatif efficace. Il est à signaler, aussi que les stagiaires éprouvent également des difficultés relatives à la qualité de la conception des situations d'enseignement-apprentissage pour le contenu à faire apprendre. Ces défaillances se traduisaient par une mauvaise présentation, voire aucune, des objectifs du cycle et des buts de la séance, l'essentiel de ce qui doit être retenu par l'élève n'est pas suffisamment souligné, voire pas identifié du tout, l'enjeu éducatif n'est ou n'a jamais été précisé, la logique d'apprentissage n'est pas évoquée...

A cet égard, on peut penser que ces difficultés peuvent revenir au fait que l'EPS ne peut pas utiliser un savoir identifié, existant dans le champ social, pour construire ses contenus. D'où la difficulté pour la profession de se mettre d'accord sur un savoir commun à faire passer. L'EPS utilise donc comme support de cours, les pratiques physiques sociales existantes (sportives ou non sportives). Ces pratiques ne sont pas un savoir en soi et ne peuvent pas être enseignées pour elles mêmes, mais sont l'occasion de développer des conduites motrices et cognitives plus ou moins intelligentes et efficaces pour résoudre les problèmes dont elles sont porteuses. Ces conduites nécessitent la mise en œuvre de savoirs méthodologiques : apprendre à faire des hypothèses - à se fixer un but à identifier les sous buts - à vérifier l'efficacité de la démarche retenue - à la modifier si nécessaire en prenant en compte les résultats obtenus - à développer une attitude de métacognition, de savoirs faire et des savoirs être qui constituent pour certains les contenus de l'EPS (Paquay, 2002). De ce fait les contenus de l'EPS ne se résument pas à l'APS, ni à la technique, ni à des comportements sociaux jugés conformes à la norme, mais doivent être construit pour rendre nécessaires ces conduites intelligentes supports de ces différents savoirs. Mais devant l'urgence inhérente à toute situation d'enseignement et faute de temps et parfois de compétences pour faire face à la transmission de ces différents savoirs, la tentation est grande pour les enseignants de privilégier les savoirs être (Malet et Brisard, 2007).

Egalement, cette étude a permis de mettre l'accent sur les difficultés que ces stagiaires éprouvent au cours des actes d'interventions pédagogiques. Elles, se traduisent par une mauvaise adéquation entre les prévisions nécessaires et le matériel disponible, mauvais choix tant en ce qui concerne l'emplacement que le matériel utilisé (non adapté), la séparation, en fonction du sexe, est systématique et un peu simpliste et l'identification des formes judicieuses de groupement (affinité, besoins, motivation, niveau d'efficacité, etc.) est difficile. En effet, pour le stagiaire cela se traduit par des préparations construites indépendamment du comportement des élèves et organisées essentiellement par la logique du savoir. A l'interaction de la logique de l'apprenant et de la logique didactique l'enseignant est confronté à la gestion d'un conflit entre procédure d'apprentissage et produit de cet apprentissage (Paillard, 1990). L'apprentissage est envisagé ici comme le 
développement d'une activité adaptative, visant une transformation pertinente et durable, dans leur nature et dans leur structure, des comportements de l'élève. Le savoir à construire comme produit attendu de l'apprentissage doit être mis en relation avec la dimension méthodologique inhérente à tout apprentissage. Cette dimension doit être envisagée comme l'articulation des différentes opérations mentales de régulation de l'activité adaptative. L'élève doit donc comprendre, raisonner, évaluer pour s'approprier le savoir et comprendre les raisons et les conditions de ses réussites. L'obstacle à dépasser pour résoudre ce conflit est la centration sur une conception magique de la tâche qui renvoie à une pédagogie des situations. Cette centration se traduit par une confusion entre activité/action et apprentissage. Pour l'enseignant débutant cela se traduit par une priorité à la mise en action des élèves qui s'auto-suffirait pour faire apprendre les élèves (Worthy, 2005). Cette dimension est relative à l'aspect psycho-sociologique de l'enseignant. Elle est au croisement de deux logiques qui parfois s'affrontent. Une logique sociale qui conduit à la formation de professionnels à qui la société donne mission d'enseigner pour faire apprendre tous les élèves. Les programmes, la structure de l'école, son environnement social et culturel détermine un espace dans lequel le futur enseignant doit s'insérer pour y peser. Cette nécessaire insertion peut buter de manière problématique sur la logique psychologique du stagiaire. Sa personnalité, ses projets personnels, ce qu'il est, ses attitudes, ses convictions, constituent autant d'éléments en jeu dans le processus de formation (Perrenoud, 2001). C'est dans ces rapports contradictoires qu'il s'agira de construire les attitudes, les comportements et une certaine éthique qui vont lui permettre d'assurer et d'assumer sa place dans le système éducatif. Il en va ainsi par exemple des compétences, des attitudes qui vont lui permettre l'autorité, la gestion des conflits, le travail en équipe.

Cette dimension est peu prise en compte dans les formations professionnelles des enseignants. Elle est laissée en gestion autonome par le sujet objet de la formation et l'institution livre la construction de cette dimension au hasard des interactions avec le terrain, sachant que ces interactions peuvent être plus ou moins heureuses (Leclerc, 2004).

La dimension psychologique, l'accompagnement de la personne dans la construction de son identité professionnelle dans les instituts de formation professionnelle relève donc de la formation sur le tas. Cette non prise en compte peut par moments être source de difficultés pour les individus en formation et mettre à mal le processus de formation professionnelle. Par conséquent, le stagiaire reste profondément influencé par des notions ou des images qui lui viennent d'un vécu bien antérieur à sa période de formation (Carlier, Cloes, Gaspard, Gérard, Laraki, Lenzen, et Piéron, 2002). Pour récapituler, nous signalons que la logique du développement résulte de l'activation des processus de l'étudiant, celui de la prise de connaissance sur son objet et passe par un dispositif avec des aides et des contraintes pour que l'étudiant puisse à partir des difficultés éprouvées identifier les problèmes sous-jacents, les déséquilibres en mettant en relation ce qu'il découvre au niveau de son objet et ses façons de faire. On est donc tout à fait d'accord avec Schön (1983) quand il parle de problem setting à la place de problem solving. Néanmoins, il est à signaler que par la même occasion Schön (1982) indique que les universités ne se consacrent pas suffisamment à l'élaboration et à la transmission d'un savoir fondamental en général. Ce sont les institutions qui adoptent en grande partie une épistémologie particulière, une vision tronquée de la connaissance, nourrie par un manque d'attention sélective à la compétence pratique et à l'art du professionnel. Pour Martinez (1993), il est nécessaire d'utiliser plusieurs types de procédures pour provoquer et entraîner les étudiants à réfléchir sur leur pratique, en stimulant les deux processus articulés de prise de connaissance et de prise de conscience (Piaget, 1974).

Finalement et au-delà des contenus à enseigner à nos étudiants, futurs enseignants (le quoi), n'est-il pas nécessaire de nous interroger sur nos procédures de formation (sur le comment)?

\section{Conclusion}

L'objectif de la présente étude consistait à évaluer la qualité de la formation des enseignants d'éducation physique et sportive dans les ISSEP Tunisiens. Plus précisément l'appréciation de la qualité d'appropriation des compétences professionnelles lors du stage de préparation à la vie professionnelle. Ce stage qui s'étale sur toute l'année scolaire est d'une durée de 104 heures. Il s'effectue sous le tutorat de l'enseignant formateur et du maître de stage. L'observation didactique a porté sur 100 séances d'EPS animées par les étudiants stagiaires. Cinq compétences ont été fixées pour cette étude : La communication dans la langue d'enseignement, la conception des situations d'enseignement et d'apprentissage, le pilotage des situations d'enseignement et d'apprentissage, l'évaluation de la progression des apprentissages et agir de façon éthique et responsable dans l'exercice de la fonction enseignante. Ces compétences ont fait l'objet d'une évaluation critériée à travers des indicateurs qui leur sont spécifiques. L'analyse des différents résultats de l'observation didactique a permis de déduire qu'une réflexion sur les conditions de l'intégration efficace et efficiente des contenus de formation sera poursuivie en relation avec les données de l'ergonomie et les recherches les plus actuelles notamment. Les formateurs doivent impérativement participer à l'évaluation des effets de la formation et contribuent à la construction de l'image de l'état des pratiques et à la dynamique de la réflexion professionnelle nécessaire à l'accroissement de l'efficacité et de son élargissement. La part disciplinaire de la 
formation actuelle s'attache à être en prise sur les savoirs professionnels directement investis dans les pratiques de classe. En effet, les stagiaires devront pouvoir être en mesure de reconnaître les éléments de leur pratique et situer l'état de leurs compétences actuelles au regard des différentes dimensions de leur mission et des exigences de l'enseignement des programmes. Ils devront pouvoir mettre à jour leurs compétences didactiques par l'intégration des problématiques actuelles : organisation de formes de pratiques scolaires des APS, animation interactive des différents rôles sociaux associés, aide permanente et renforcée à l'apprentissage, évaluation des compétences. Egalement, ces stagiaires devront pouvoir acquérir les connaissances nécessaires au développement de la réflexivité nécessaire à la poursuite de leur développement professionnel.

Pour cela, une approche réellement contractualisée devra être mise en oeuvre. Elle supposera un engagement dans la durée des stages de préparation à la vie professionnelle et des formes de travail mobilisant la construction collective de compétences et le partage des savoirs professionnels (Altet, 1994). Bien évidemment, le travail sur les pratiques réelles devrait être central. Au terme de la formation initiale, les nouveaux enseignants ne se sentent pas toujours prêts pour affronter le marché du travail et peuvent vivre diverses difficultés. Néanmoins, comme nous l'avons vu précédemment, plusieurs auteurs croient que c'est avec l'expérience que l'enseignant débutant parviendra à développer de meilleures compétences en tant qu'enseignant. Plusieurs possibilités s'offrent aux enseignants nouvellement recrutés afin d'acquérir de savoir plus actualisé et de nouvelles compétences et connaissances, notamment la pratique réflexive individuelle ou en interaction avec les pairs, les programmes d'insertion professionnelle et la formation continue.

\section{Références}

[1]. Albero, B. (2000). L'autoformation en contexte institutionnel. Paris, France : L'Harmattan.

[2]. Altet, M. (1994). La formation professionnelle des enseignants. Paris, France : Presses universitaires de France.

[3]. Astolfi, J-P. (2003) ; Savoirs en action et acteurs de la formation. Rouen, France : Publications universitaires de Rouen.

[4]. Barbier, J. M. (1985) L'évaluation en formation. Paris, France : Presses universitaires de France.

[5]. Bourdoncle, R. et Lessard, C. (2003). Qu'est-ce qu'une formation professionnelle universitaire ? Les caractéristiques spécifiques : programmes, modalités et méthodes de formation. Revue Française de Pédagogie, $142,131-181$

[6]. Bellier, S. (2000). Compétences en action, expérimentations, implications, réflexions pratiques. Paris, France : Editions Liaisons.

[7]. Brauantony, S. et Christine, B. (2005). Réflexivité et pratiques de formation. Regards critique. Revue carrefours de 1'éducation, 20, 113-122.

[8]. Brown, JS.S. et Duguid, P. (1989). Situated cognition and the culture of learning. Educational Researcher, 18, 32-42

[9]. Carre, P. et Caspar P. (2002). Traité des sciences et des techniques de la formation. Paris, France : Editions Dunod.

[10]. Carre, P. et Moisan, A. (2002). La formation autodirigée. Aspects psychologiques et pédagogiques. Paris, France : L’Harmattan.

[11]. Carlier, G., Cloes, M., Gaspard, L., Gérard, Ph., Laraki, N., Lenzen, B., Piéron, M. et Renard, J. P. (2002). Adéquation entre les formations existantes en éducation physique, les motivations des étudiants et les différents débouchés professionnels. Etude réalisée à la demande de la Ministre Fr. Dupuis, UCL : Louvain-la-Neuve. ULg, Liège.

[12]. Cauterman, M. M., Demailly, L., Suffys S. et Bliez-Sullerot, N. (1999). La formation continue des enseignants est-elle utile ? Paris, France : Presses universitaires de France.

[13]. Clancey, W. J. (1991). Situated cognition: stepping out of representation flatland. A response to Swann's commentary. AICOM, 4, 109-112.

[14]. Famose, J. P, Hébrard, A., Simonet, P et Vives, J. (1979). Contribution de l'"aménagement matériel du milieu" à la pédagogie des gestes sportifs individuels. Compte rendu de fin d'étude d'une recherche financée par la D.G.R.S.T. Paris, France : institut national du sport et de l'éducation physique.

[15]. Famose, J. P. (1983). Stratégies pédagogiques, tâches motrices et traitement de l'information. Revue EPS, 13.

[16]. Gentile, A. M. (1972). A working model of skill acquisition to teaching. Quest, 17, 3-23.

[17]. Gomez, F. (2004). Le perfectionnement professionnel : essai de construction d'un objet de recherche. Education permanente, 161.

[18]. Guskey, T. R. (2000). Evaluating Professional Development. Thousand Oaks CA : Corving Press.

[19]. Hadji, C. (1989). L'évaluation, règles du jeu. Des intentions aux outils. Paris, France : ESF.

[20]. Huberman, M. (1992). De la recherche à la pratique: comment atteindre des retombées fortes ? Revue Française de Pédagogie, 98, 515 .

[21]. Leclerc, C. (2004). Le formateur d'enseignants : entre directivité et réflexivité. Dans J. P.

[22]. Martinez, C. (1993). La compétence à enseigner les activités physiques et sportives : une série de couples ago-antagonistes. Actes du Deuxième Congrès Européen de Systémique. Prague, volume I, 35-44.

[23]. Maroy, C. (2005). Les évolutions du travail enseignant en Europe. Facteurs de changement, incidences et résistances. Les cahiers de la recherche en éducation et formation, 42, 4-28.

[24]. Malet R. et Brisard E. (2007). L'accompagnement des enseignants sur le terrain saisi par les acteurs de la formation. Revue de l'ADMEE : Mesure et évaluation en éducation, 1, 55-78.

[25]. Medely, D.M. (1972). Early history of research on teacher behavior. International Review of Éducation, 18, $34-42$.

[26]. Paquay, L. (2002). Formateurs d'enseignants. Quelle professionnalisation? Bruxelles, Louvain-la-Neuve : Edition De Boeck.

[27]. Paillard, J. (1990). Réactif et prédictif : deux modes de gestion de la motricité. Dans V. Nougier et J.R Blanqui (Dir.) : Pratiques sportives et modélisation du geste. Grenoble : Collection Grenoble Sciences.

[28]. Pastré, P. (2002). L'analyse du travail en didactique professionnelle. Revue française de pédagogie numéro, 138,9 -17.

[29]. Perrenoud, P. (2001). De la pratique réflexive au travail sur l'habitus. Recherche et formation, 36, 131-162.

[30]. Piaget, J. (1974). Réussir et comprendre, Paris, France : Presse universitaires de France.

[31]. Rayou, P. et Van Zanten, A. (2004). Enquête sur les nouveaux enseignants. Changeront-ils l'école ? Paris, France : Bayard.

[32]. Rayou, P., Gélin, D. et Ria, L. (2007). Entrer dans le métier d'enseignant. Paris, France : Armand Colin.

[33]. Romainville, M. (2002). L'évaluation des acquis des étudiants dans l'enseignement universitaire. Paris, France : Presses universitaires de France.

[34]. Ria, L., Sève, C., Theureau, J., Saury, J. et Durand, M. (2003). Beginning teacher's situated emotions : study about first classroom's experiences. Journal of Education for Teaching, 29, 219-233. 
[35]. Roux-Perez, T. (2004). L'identité professionnelle des enseignants d'EPS : entre valeurs partagées et interprétations singulières. Revue science et technique des activités physiques et sportives, 63, 75-88.

[36]. Samurcay, R. et Pastre, P. (1995). Outiller les acteurs de la formation pour le développement des compétences. Education permanente, $123,13-31$

[37]. Schön, D. (1983). Le praticien réflexif. Montréal : Editions Logiques.

[38]. Shiffrin, R.M. et Schneider, W. (1977). Controlled and automatic human information processing : Perceptual learning, automatic attention and a general theory. Psychological Review, 84, 1-66.

[39]. Suchman, L. A. (1987). Plans and situated actions : the problem of human-machine communication. Editeur : Cambridge University Press.

[40]. Shulman, L. S. (1987). Knowledge and teaching: foundations of the new reform. Harvard Educational Review, 57, 65-78.

[41]. Theureau, J. (2000). Anthropologie cognitive et analyse des compétences. Paris, France : Presses universitaires de France.

[42]. Vygotski, L. (1997). Pensée et langage. Paris, France : Presses universitaires de France.

[43]. Worthy, J. (2005). It didn't have to be so hard : the first years of teaching in an urban school. International Journal of Qualitative Studies in Education, 18, 379-398.

Tableau 1

Descriptif des différentes activités physiques enseignées

\begin{tabular}{ccccccc}
\hline Type d'APS & \multicolumn{3}{c}{ Sports individuels } & \multicolumn{2}{c}{ Sports collectifs } \\
\hline & Course de & Saut en & Lancer du & Course de & Basket & Handball \\
& vitesse & longueur & poids & relais & 7 & 15 \\
Nombre de séances & 20 & 15 & 18 & 7 & 25 \\
\hline
\end{tabular}

Tableau 2

Données relatives à la qualité d'appropriation des compétences professionnelles

\begin{tabular}{llc}
\hline & $\mathbf{Z}$ & $\sigma$ \\
\hline Compétence 1 & 13.34 & 1.95 \\
Compétence 2 & 12.05 & 1.98 \\
Compétence 3 & 13.11 & 2.36 \\
Compétence 4 & 12.55 & 1.76 \\
Compétence 5 & 15.63 & 2.28 \\
\hline
\end{tabular}

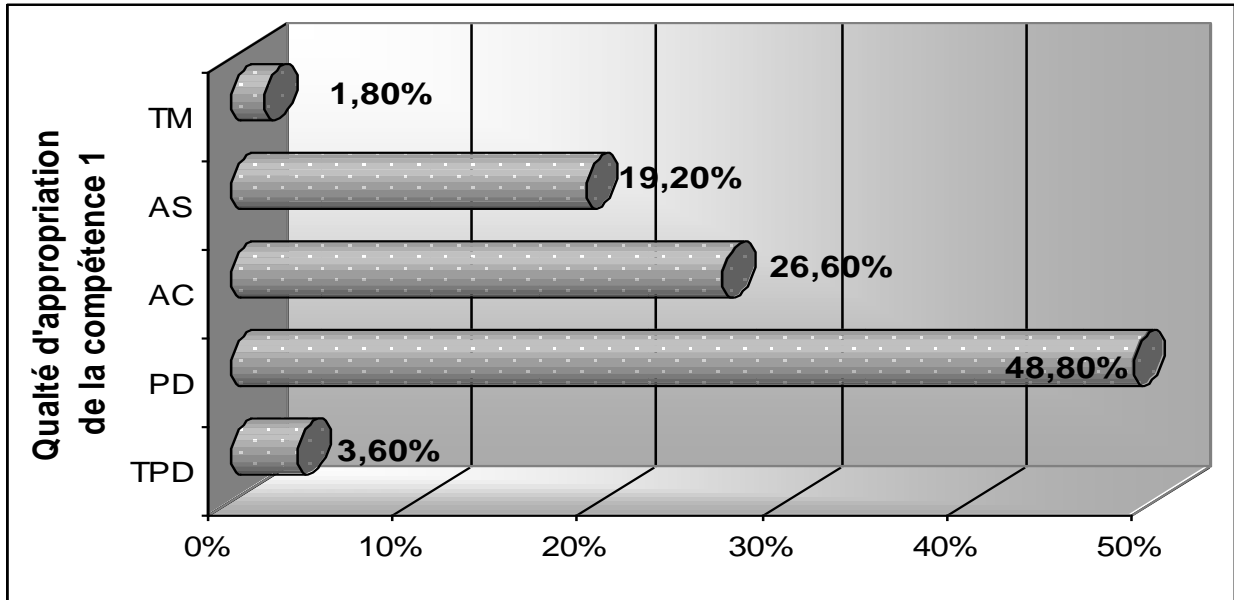

Figure1. Pourcentages relatifs à la qualité d'appropriation de la compétence 1

Tableau 3

Qualité d'appropriation des différents indicateurs relatifs à la compétence 1

\begin{tabular}{|c|c|c|c|}
\hline Indicateurs & & $\bar{x}$ & $\sigma$ \\
\hline Utilise un langage oral varié et soigné dans ses interventions. & ind 1 & 2,51 & 0,72 \\
\hline Utilise un langage oral varié et soigné dans différents contextes. & ind 2 & 2,78 & 0,86 \\
\hline Corrige les erreurs commises par les élèves pendant l'exécution. & ind3 & 2,81 & 0,85 \\
\hline Exprime ses idées de façon claire et articulée dans divers contextes. & ind 4 & 2,54 & 0,80 \\
\hline Utilise correctement le vocabulaire technique propre à sa discipline. & ind5 & 2,70 & 1,09 \\
\hline
\end{tabular}




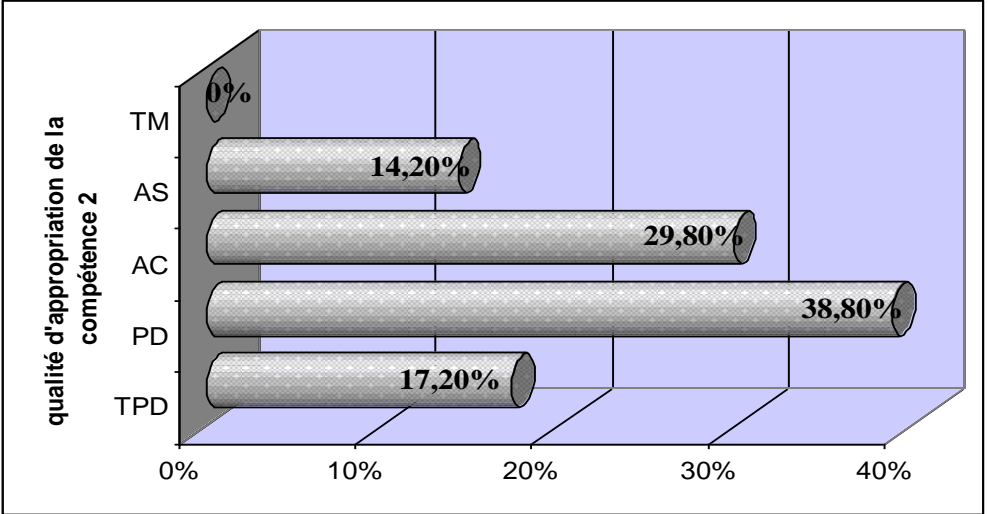

Figure 2. Pourcentages relatifs à la qualité d'appropriation de la compétence 2

Tableau 4

Qualité d'appropriation des différents indicateurs relatifs à la compétence 2

\begin{tabular}{|c|c|c|c|}
\hline Indicateurs & & $\bar{x}$ & $\sigma$ \\
\hline Prévoit des situations d'apprentissage favorisant la participation active des élèves. & ind 1 & 2,57 & 0,92 \\
\hline Prévoit des retours avec les élèves sur les apprentissages effectués. & ind 2 & 2,56 & 0,94 \\
\hline $\begin{array}{l}\text { Prévoit des organisations variées qui tiennent compte des différences individuelles des } \\
\text { élèves. }\end{array}$ & ind 3 & 2,47 & 0,83 \\
\hline Précise les critères d'évaluation. & ind 4 & 2,53 & 0,83 \\
\hline Planifie des situations motrices de consolidation supplémentaires. & ind 5 & 1,92 & 0,97 \\
\hline
\end{tabular}

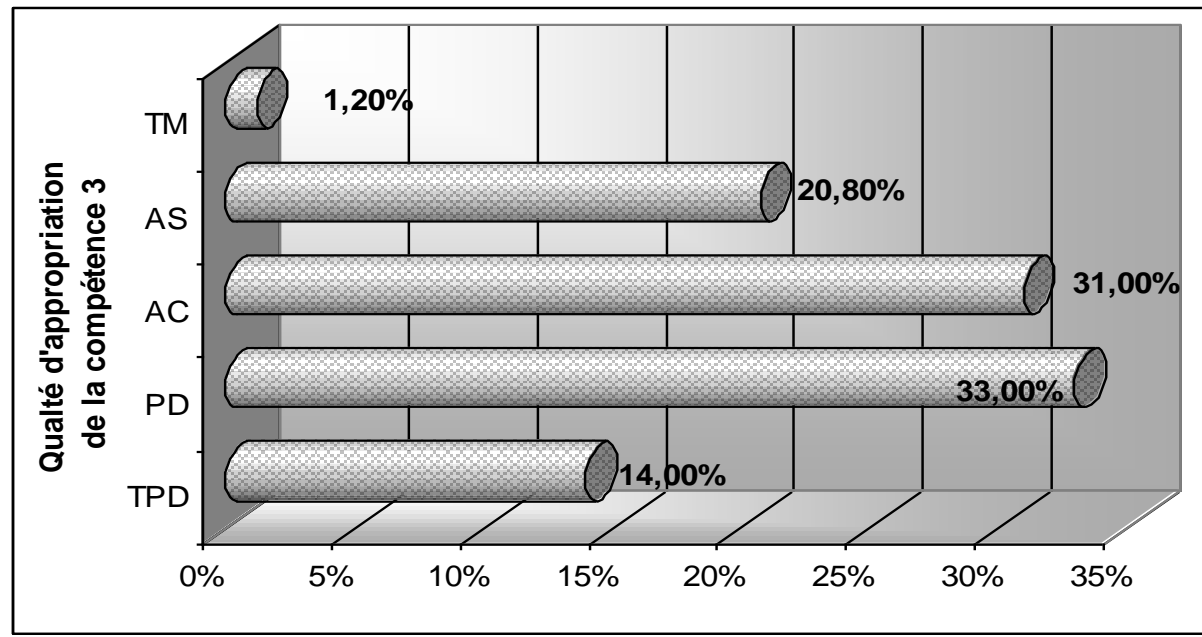

Figure 3. Pourcentages relatifs à la qualité d'appropriation de la compétence 3

Tableau 5

Qualité d'appropriation des différents indicateurs relatifs à la compétence 3

\begin{tabular}{|c|c|c|c|}
\hline Indicateurs & & $\bar{x}$ & $\sigma$ \\
\hline Explique la tâche à exécuter en utilisant la démonstration gestuelle. & ind 1 & 2,73 & 0,89 \\
\hline Précise aux élèves les ressources utiles pour effectuer les apprentissages. & ind 2 & 2,24 & 0,99 \\
\hline S’assure que les élèves sont organisés de manière adéquate. & ind 3 & 2,85 & 0,89 \\
\hline Encourage les élèves par des gestes discrets ou par des paroles. & ind 4 & 2,86 & 0,95 \\
\hline Respecte les intentions prévues dans sa planification. & ind 5 & 2,43 & 1,12 \\
\hline
\end{tabular}




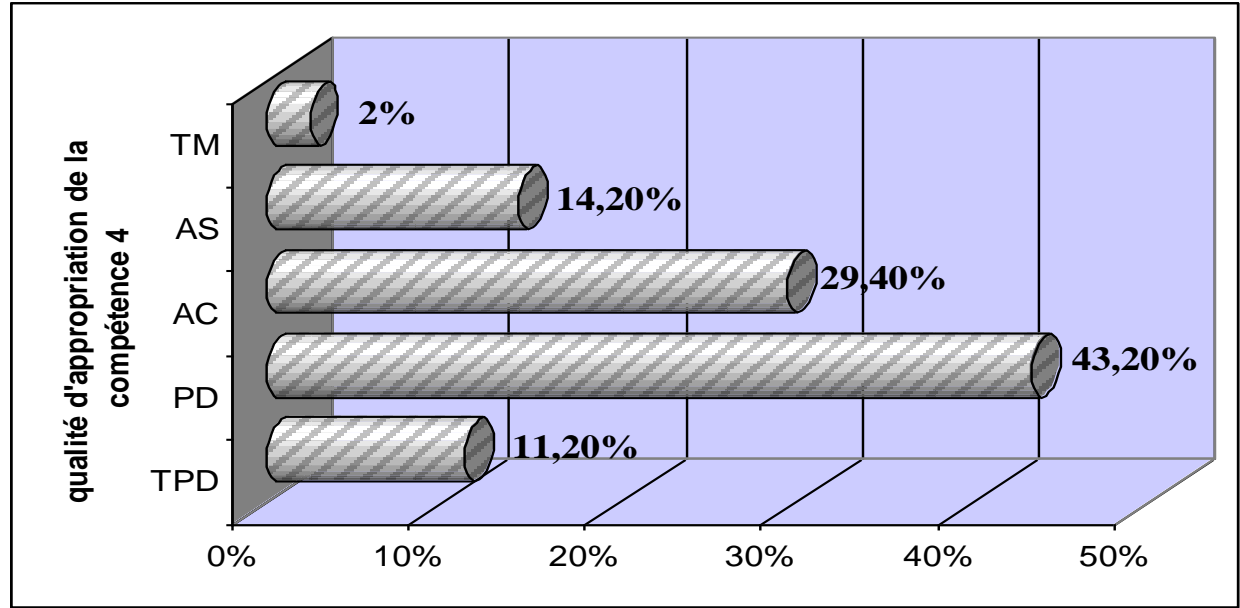

Figure 4. Pourcentages relatifs à la qualité d'appropriation de la compétence 4

Tableau 6

Qualité d'appropriation des différents indicateurs relatifs à la compétence 4

\begin{tabular}{lccc}
\hline \multicolumn{1}{c}{ Indicateurs } & & $\bar{x}$ & $\sigma$ \\
\hline Utilise des moyens didactiques permettant d'évaluer la progression des & ind 1 & 2,46 & 1,13 \\
apprentissages des élèves. & & & \\
Donne des rétroactions aux élèves. & ind 2 & 2,68 & 0,86 \\
Fait un bilan des apprentissages effectués par les élèves. & ind 3 & 2,71 & 0,80 \\
Soutient l'initiative des élèves sur les stratégies d'apprentissage. & ind 4 & 2,36 & 0,97 \\
Amène les élèves à expliquer leurs réussites ou leurs difficultés. & ind 5 & 2,34 & 0,81 \\
\hline
\end{tabular}

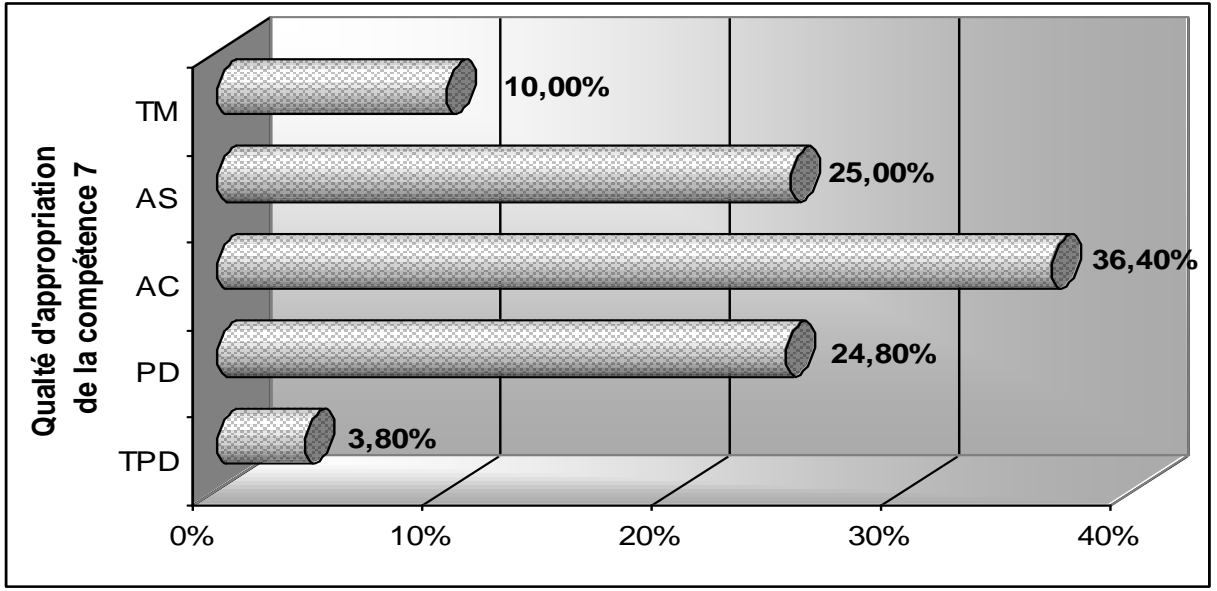

Figure 5. Pourcentages relatifs à la qualité d'appropriation de la compétence 5

Tableau 7

Qualité d'appropriation des différents indicateurs relatifs à la compétence 5

\begin{tabular}{lcc}
\hline \multicolumn{1}{c}{ Indicateurs } & $\overline{\mathcal{C}}$ & $\sigma$ \\
\hline Agit d'une manière démocratique dans sa classe ou dans les ateliers. & ind 1 & 3,04 \\
Evite toute forme de discrimination envers les élèves, les parents et ses pairs. & ind 2 & 3,16 \\
Maintient une attitude positive envers tous ses élèves. & ind 3 & 3,89 \\
Maintient une attitude positive envers ses collègues. & ind 4 & 0,95 \\
Maintient une attitude positive envers la direction d'établissement. & ind 5 & 3,30 \\
\hline
\end{tabular}

